\title{
Polymorphic transition of tin under shock wave compression: Experimental results
}

\author{
C. Chauvin, J. Petit, and F. Sinatti \\ Comissariat à l'Énergie Atomique et aux Énergies Renouvelables, Centre de Gramat, BP. 80200, \\ 46500 Gramat, France
}

\begin{abstract}
In this work, the $\beta$-bct polymorphic transition in tin is investigated by means of plate impact experiments. The $\mathrm{Sn}$ target surface is observed in a partially released state obtained thanks to a transparent lithium fluoride (LiF) anvil. We report both measurements of interface velocity and temperature obtained using Photon Doppler Velocimetry and IR optical pyrometer on shock-loaded tin from 8 to $16 \mathrm{GPa}$. We show that the Mabire Model EOS associated to the SCG plasticity model provides an overall good estimate of the velocity profiles. However, depnding on the shock amplitude, its prediction of the temperature profile may be less satisfactory, hence underlining the need for future improvements in terms of phase transition kinetics description.
\end{abstract}

\section{Introduction}

During the last ten years, breakthrough has been achieved in terms of modeling phase transition of metals under shock loading. For example, the multiphase EOS developed by C. Mabire [1] has been successfully applied to the restitution of phase change in tin. However, the capabilities and limitations of this kind of model remain partially unknown, in particular due to lack of reliable reference temperature data.

This paper presents some experimental results on tin from 8 to $16 \mathrm{GPa}$ around the polymorphic transition under dynamic loadings. Interface velocity and temperature measurements at $\mathrm{Sn} / \mathrm{LiF}$ allow to show the tin transforms from $\beta$ phase to bct phase.

Additionnaly, this paper presents a comparison between the numerical and experimental interface velocity and interface temperature taking into account the heat transfer phenomena.

\section{Experiments}

The tin used in these experiments had a purity of 99.9 $\%$ and a density of $7.287 \mathrm{~g} / \mathrm{cm}^{3}$. The material target were polished to present a surface of high optical quality roughness $\left(\mathrm{R}_{\mathrm{a}}<20 \mathrm{~nm}\right)$.

To improve our understanding of the polymorphic transition phenomenon and to investigate the phase diagram, both measurements of interface velocity and temperature are achieved. But, the experimental measurements must be very reliable to be compared with our multiphase EOS.

The diagnostic of PDV allow to obtain the velocity with a satisfying precision $(<1 \%)$. But, deducing a dependable temperature from the signals provided by a high-speed two-wavelength infrared optical pyrometer is still complex particularly at these low temperatures.

\subsection{Temperature measurement theory}

High-speed multi-wavelength infrared optical pyrometry is the most effective diagnostic to achieve low temperature measurement below $1000 \mathrm{~K}[2,3]$. Although the method to collect radiance is well known, measurement at these low temperatures is still more complicated due to the low amplitude of the radiated energy: any potential background lights must be prevented and generally avoided and under control.

But the technical challenge of this measurement is to deduce from the collected radiance an accurate interface temperature depending on the evaluation of the dynamic emissivity. Because, the simultaneous measurement of emissivity of the thermally radiating shocked surface is still difficult to measure [4,5], a way to overcome the lack of knowledge of emissivity consists in artificially increasing the dynamic emissivity of the material up to an apparent value as close as possible to that of 1 (the blackbody emissivity) [6]. The detected radiance is then amplified, the range of dynamic emissivity is restricted and so the interface temperature becomes accurate.

\subsubsection{Using an emissive layer at interface}

To measure low temperature $(<800 \mathrm{~K})$ under dynamic loading, a multi-wavelength pyrometer is the common diagnostic. Its principle relies on the collect of the light generated by the material under shock loading with infrared InSb detectors which convert it a voltage signal at several wavelengths (details of the pyrometer can be found in [6]). In our experiments, the radiance comes from the surface of the material through an anvil window LiF preventing the fully release of the material. Thanks to a calibration, the conversion between measured voltages and interface radiances is determined, however inferring an interface temperature is not straightforward due to the lack of knowledge about the dynamic emissivity. The method [7] consists in:

- constraining the possible variation of the emissivity between two extreme values from its static emissivity and 1 (the emissivity is supposed increasing under loading due to degradation of the surface), 

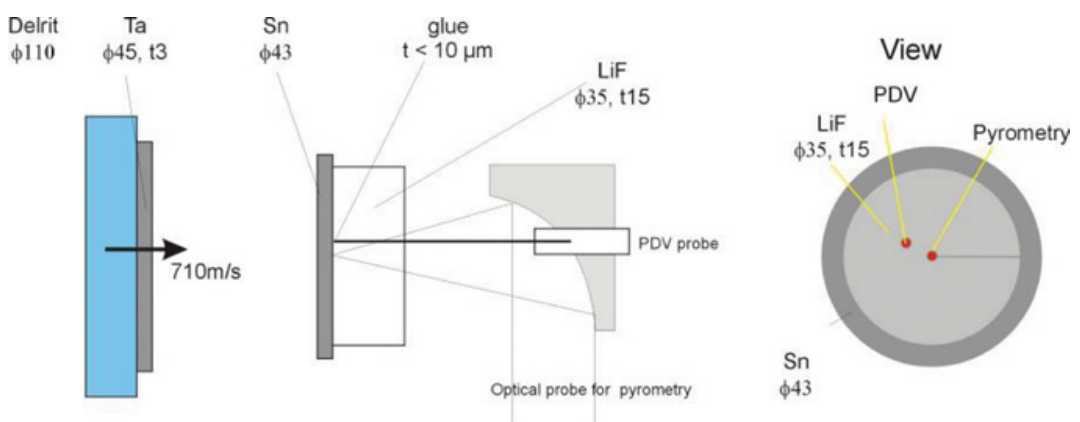

Fig. 1. Experimental setup.

- and applying these values at the different wavelength channels of pyrometer, an uncertainty range of temperature in which the true temperature is determined. Generally the shortest wavelength (channel 2 in this study) which is the less sensitive to the emissivity gives the more restricted temperature range. The other wavelengths help to evaluate the evolution of the emissivity.

A way to restrain the bounds of emissivity is to increase artificially the material emissivity up to an apparent value as close as possible to that of a blackbody applying an emissive layer. The range of dynamic emissivity is then restricted and so the uncertainty on interface temperature [6].

\subsubsection{Inferring the material temperature}

Besides the difficulty in deducing an accurate temperature measurement, assessing to the material temperature needs to understand the heat transfer phenomena at interface. Between two adjacent materials under dynamic loading, only thermal conduction provides heat transfer. Thanks to Fourier's law, interface and material temperatures can be linked depending on thermal properties of each material [8]:

$$
T_{\mathrm{int}}=T_{1}-\frac{T_{1}-T_{2}}{1+\alpha} \text { with } \alpha=\frac{\sqrt{\left(k \rho C_{V}\right)_{1}}}{\sqrt{\left(k \rho C_{V}\right)_{2}}}
$$

where $k$ is the thermal conductivity, $\rho$ the density and $C_{V}$ the specific heat, $\mathrm{T}_{1}, \mathrm{~T}_{2}, \mathrm{~T}_{\mathrm{int}}$ the temperatures of the two adjacent materials and the interface temperature.

Considering an interface with a glue layer, the $\alpha$ value estimated from the static value is largely over 18 whatever the metallic material, so the interface and material temperatures are almost identical showing the advantage of a glue layer due to its insulator properties.

At a complex interface and particularly with the presence of an emissive layer and glue layer, the thermal conductivity of the emissive layer must be enough important and its thickness enough thin to enable an efficient heat exchange and to obtain the temperature of the emissive layer as close as possible to that of the material. The glue layer, as for it, allows the interface temperature to be similar to the temperature of the emissive layer and so to that of the material.

With the use of a thin $\mathrm{ReSi}_{2}$ layer at interface and thanks to its efficient thermal-mechanical properties, the
Table 1. Parameters for the impact experiments on tin.

\begin{tabular}{|c|c|c|c|}
\hline & $\begin{array}{c}\text { Target } \\
\text { thickness }(\mathrm{mm})\end{array}$ & $\begin{array}{c}\text { Impact } \\
\text { velocity }(\mathrm{m} / \mathrm{s})\end{array}$ & $\begin{array}{c}\text { impact stress } \\
(\mathrm{GPa})\end{array}$ \\
\hline Sn01 & 2 & $802.5 \pm 3.3$ & $15.2 \pm 0.5$ \\
\hline Sn02 & 2 & $704.5 \pm 0.5$ & $12.9 \pm 0.1$ \\
\hline Sn03 & 2 with a 2- $\mu$ m EL & $704.1 \pm 6.3$ & $12.9 \pm 0.7$ \\
\hline Sn04 & 3 & $555.6 \pm 2.4$ & $9.1 \pm 0.5$ \\
\hline Sn05 & 3 with a 215-nm EL & $543.2 \pm 5.5$ & $8.9 \pm 0.7$ \\
\hline Sn06 & 2 & $462.5 \pm 2.5$ & $7.9 \pm 0.3$ \\
\hline
\end{tabular}

measured temperature is close to that of the material, which is in fact the value to be identified and its uncertainties have been also significantly reduced and can be used to compare our results to a calculation.

\subsection{Experimental configuration}

Six plate impact experiments (Fig. 1) were performed to observe the behaviour of tin [9] under shock wave compression and release around its $\beta$-bct polymorphic transition at different impact velocity (Table 1).

The effect of an emissive layer (EL) on the temperature measurement at the interface $\mathrm{Sn} / \mathrm{LiF}$ has been studied to explore the role of this layer: the increase of the emissivity and the effect of thermal conduction.

\section{Experimental results}

The temperature and velocity profiles are presented in Fig. 2.

It is shown that interface velocity profiles clearly indicate direct $\beta$-bct polymorphic transition consistent with the expected compression path. For the shot $\mathrm{Sn} 02$ and $\mathrm{Sn} 03$, three distinct waves compression appeared typical of the transition: the first corresponds to the elastic precursor, the second to the plastic wave of the $\beta$ phase and the third to the plastic wave of the bct phase.

During the release, a singularity is noticed displaying the reverse transition from bct to $\beta$. The Sn01 experimentation presents a single shock wave in compression: the impact stress is too high to observe the double wave. During the release, the reverse transition is observable indicated by the presence of a singularity. For the experimentations $\mathrm{Sn} 04$ and $\mathrm{Sn} 05$ which are chosen on the $\beta$-bct 


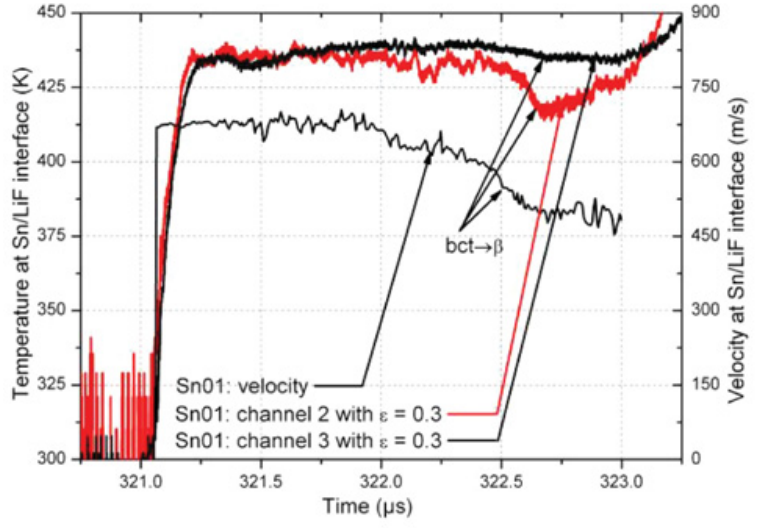

$\mathrm{Sn} 01: \mathrm{Sn} / \mathrm{LiF}$ at $11.1 \mathrm{GPa}$

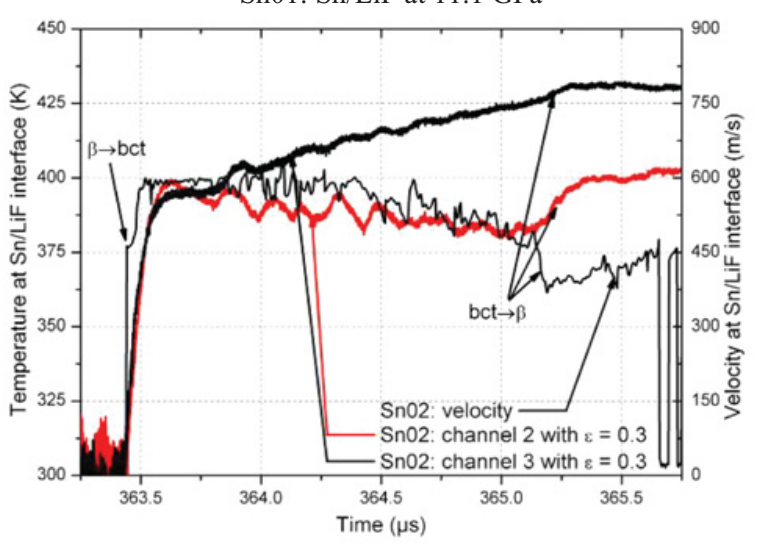

$\mathrm{Sn} 02: \mathrm{Sn} / \mathrm{LiF}$ at $9.6 \mathrm{GPa}$

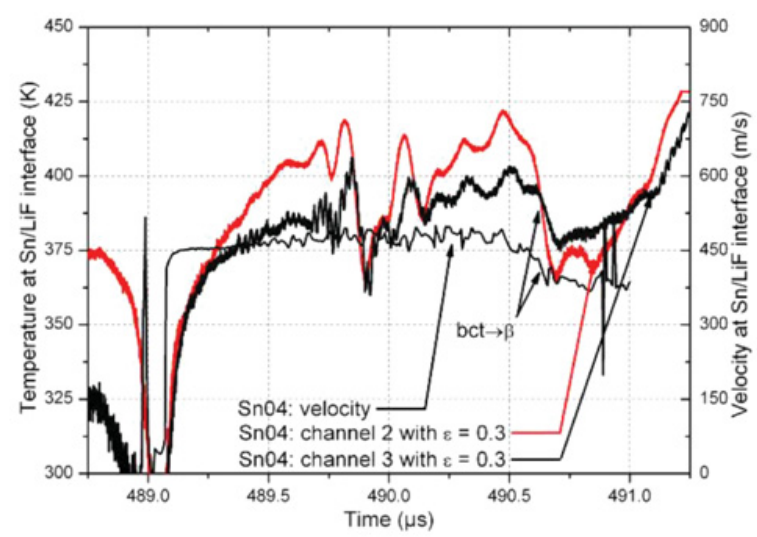

Sn04: $\mathrm{Sn} / \mathrm{LiF}$ at $7.2 \mathrm{GPa}$
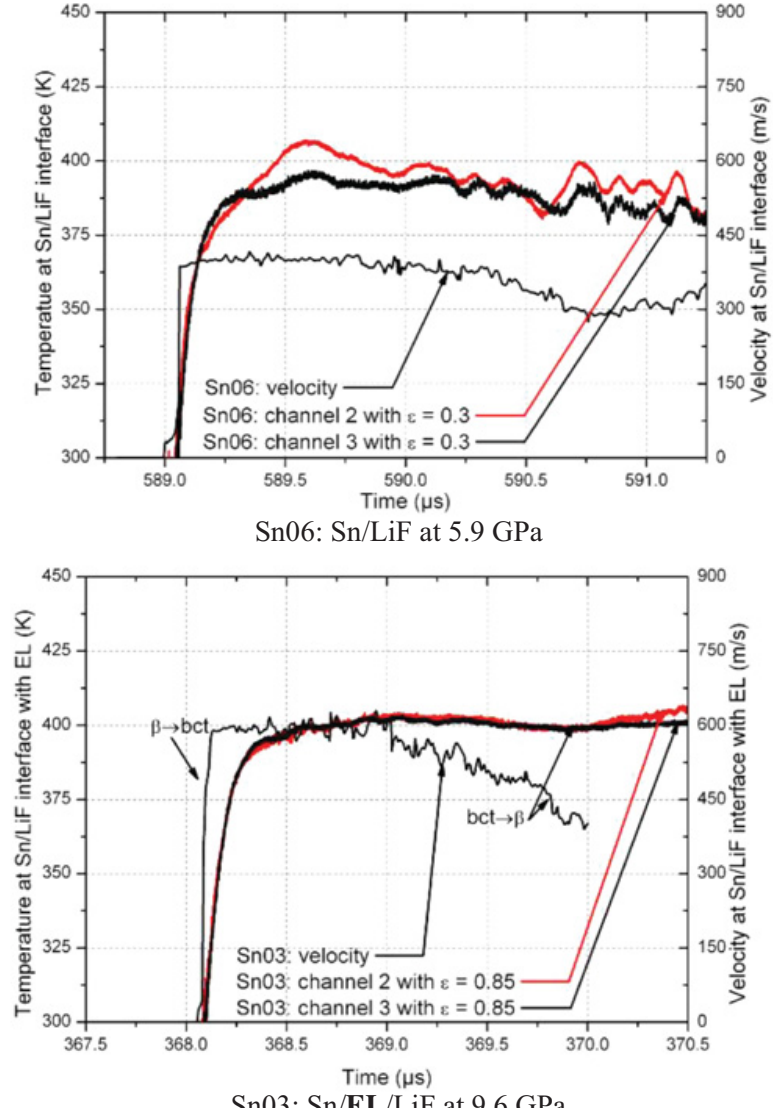

$\mathrm{Sn} 03: \mathrm{Sn} / \mathbf{E L} / \mathrm{LiF}$ at $9.6 \mathrm{GPa}$

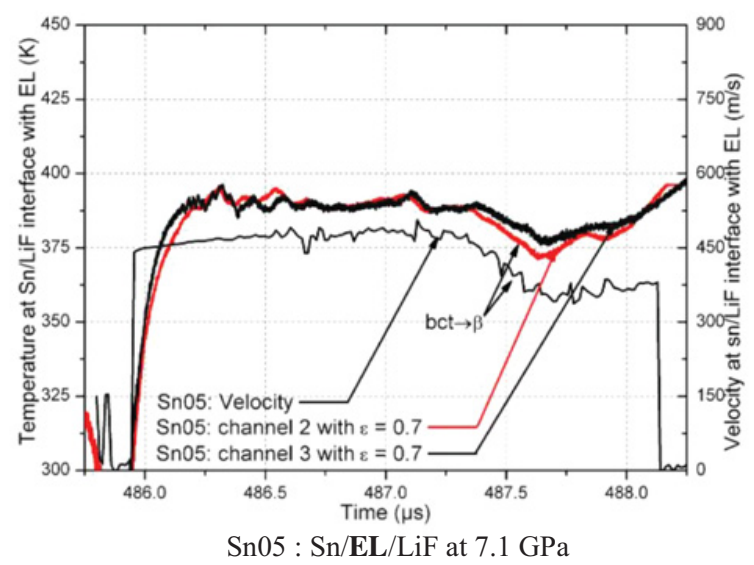

$\mathrm{Sn} 05: \mathrm{Sn} / \mathbf{E L} / \mathrm{LiF}$ at $7.1 \mathrm{GPa}$

Fig. 2. Interface temperature and velocity diagrams for the impact experiments on Tin.

frontier, no polymorphic transition is observed. In the last experimentation Sn06, the tin stays only in $\beta$ phase, so no distinctive feature indicates a polymorphic transition.

The temperature profiles at interface are presented in Fig. 2. The temperature at the interface between tin and anvil is described for each channel of the pyrometer with a constant emissivity equal to the static emissivity of the tin or equal to the static emissivity of the EL in the case of the presence of an EL at the interface.

As mentioned for the velocity profiles, the temperature shapes show distinct features function of the impact stress consistent with the compressive path. A plateau of temperature is observed during the supported shock except for the experimentations $\mathrm{Sn} 02$ and Sn04. The reverse transition is clearly noticeable as well during the release: a change of slope appears may be due to a variation of the emissivity. However, the double structure of the direct polymorphic transition is not precisely detectable, might be due to the response time of the pyrometer.

As noticed on the temperature on each channel, around the double structure of the transition for $\mathrm{SnO2}$ and $\mathrm{Sn04}$, inferring the temperature from the collected radiance with a constant emissivity is not satisfactory: the emissivity of the tin seems to change under its phase transition so the temperatures of both channels present different behaviour which could indicate the presence of a mixture of the two phases or a degradation of the surface roughness. These signals could not be considered to deduce an accurate result without difficulty. To overcome the possible variation of the emissivity, an emissive layer has been 


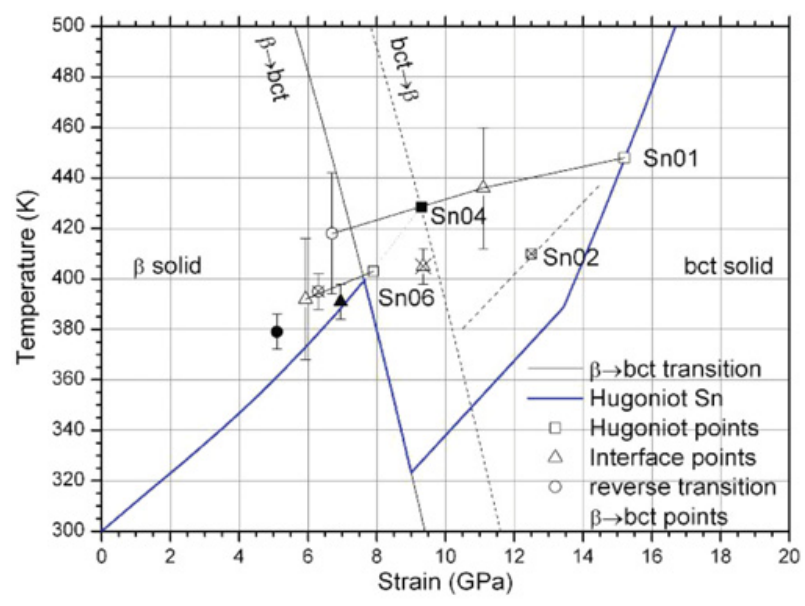

Fig. 3. Thermodynamic path of tin for the impact $\mathrm{Sn} 01, \mathrm{Sn} 02$, Sn04 and Sn06 with experimental data plotted on the phase diagram.

incorporated at the interface on the experimentations $\mathrm{Sn} 03$ and $\mathrm{Sn} 05$ allowing to obtain a profile easy to interpret and to determine a temperature with a satisfying precision: the presence of a plateau emphasize the role of an EL which seems to avoid the effects of the $\beta$-bct transition on the surface roughness or on the variation of emissivity.

The profiles in velocity and temperature reveal coherent singularities during the loading and unloading indicating the polymorphic transition of the tin.

\section{Analysis of the polymorphic transition $\beta$ phase to bet phase}

To describe the phase-diagram of tin, each $\beta$ and bct phases are represented by a Mie-Grüneisen equation of state with parameters already determined in a previous study to reproduce static and dynamic data [1]. The phase boundary is determined by the equality of the Gibbs free energy.

The singularities in the loading and unloading path are illustrated in the Fig. 3. in the temperature-strain plane. The Hugoniot states, the interface states and the singularities during the release are represented respectively by squares, triangles and circles obtaining with the experimental data.

The double structure of the wave during the loading noticed on the experimentations Sn02, Sn03, Sn04 and Sn05 reveals an hysteresis of the transition from the $\beta$ phase to bct phase, because the Hugoniot states (squares points) are beyond the static boundary. Similarly, the reverse polymorphic transition bct to $\beta$ during the release displayed on Sn01, Sn02, Sn03, Sn04 and Sn05 temperature and velocity profiles clearly indicates an hysteresis, because these singularities (circle points) are above the transition boundary and decrease as a function of stress.

For the Sn06 experimentations, the value of temperature and velocity show that tin remains in the solid $\beta$ phase during all the loading and release. This could be confirmed by the absence of any singularities in the velocity profile.

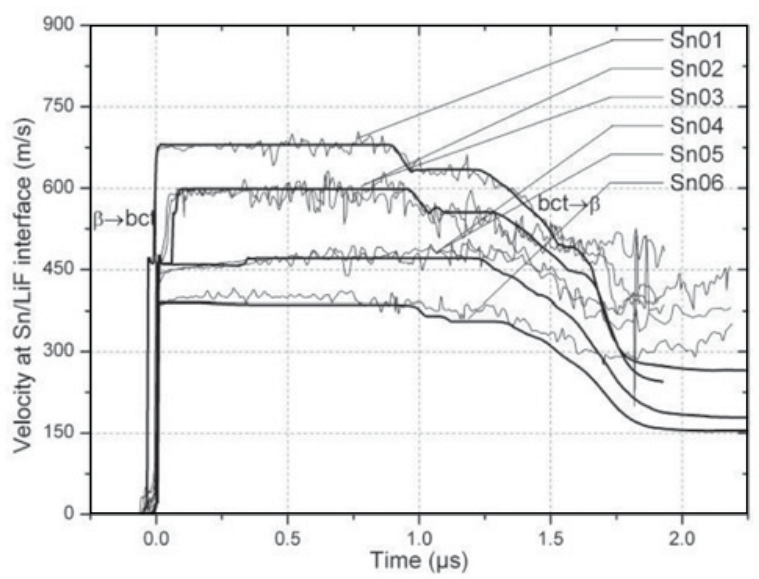

Fig. 4. Experimental and numerical comparisons for the velocity profile for Sn01, Sn02, Sn03, Sn04, Sn05 and Sn06.

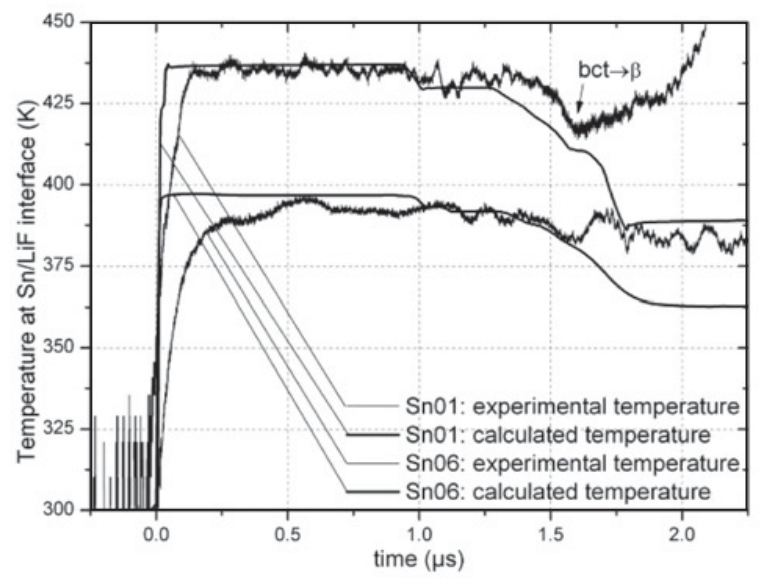

Fig. 5. Experimental and numerical comparisons for the temperature profile for $\mathrm{Sn} 01$ and $\mathrm{Sn} 06$ without an emissive layer.

Nevertheless, in the release, the temperature profile shows a change of slope which might suggest a reverse transition.

Simulations of the experiments were performed with a 1-D lagrangian code Unidim including thermal conduction modelling and the multi-phase equation of state of tin developed by Mabire [1]. The plasticity model chosen for the tin is a SCG model [10]. The comparison between experiments and calculations are illustrated in Fig. 4, Fig. 5 and Fig. 6. taking into account the hypothesis of an hysteresis during the direct and reverse transition.

Concerning the velocity profiles of all experiments (Fig. 4), the calculations accurately reproduced the direct transition $\beta$ to bet phase during the loading and the reverse transition during the unloading: the double structure of the shock wave and the singularity in the release are correctly displayed.

In Fig. 5, experimental and numerical temperatures in the Sn01 experimentation indicate the double structure in the loading and a reverse transition of tin occuring during release at about $415 \mathrm{~K}$ and these both temperatures are similar. For the Sn06 experimentation in Fig. 5, even if the level on the plateau is correctly reproduced, experimental data seems to suggest a bct- $\beta$ transition not indicated in the calculations. 


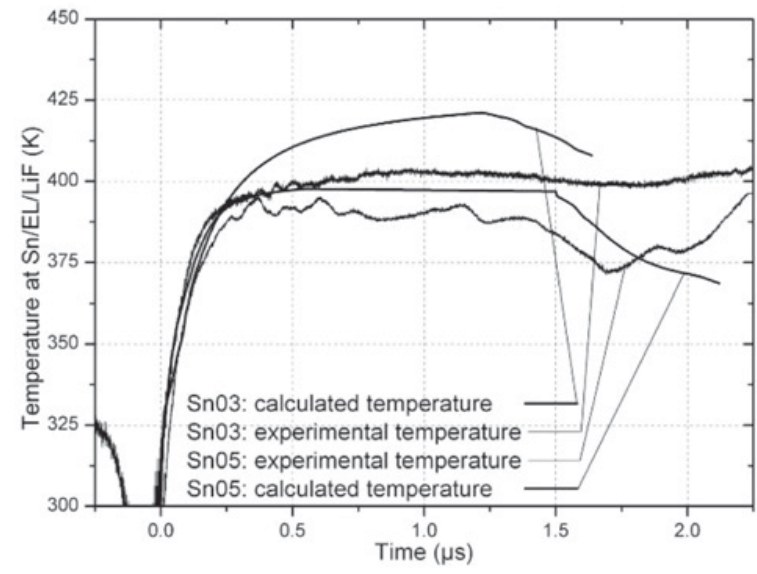

Fig. 6. Experimental and numerical comparisons for the temperature profile around the double structure of the transition for $\mathrm{Sn} 03$ and $\mathrm{Sn} 05$ with an emissive layer.

Due to the complexity to deduce a precise temperature at interface $\mathrm{Sn} / \mathrm{LiF}$ around the $\beta$-bct transition during the shock wave compression, only the temperature with an emissive layer are compared with calculated data in Fig. 6. The calculations correctly reproduce the shape. As noticed for the experimental and numerical temperatures of $\mathrm{Sn} 03$ and Sn05, a softened ramp is observed with the presence of an EL suggesting a thermal conduction effect before reaching the thermal equilibrium. This illustrates the limitation of an EL which allows to obtain an accurate experimental temperature but which prevents from displaying a $\beta$-bct transition during the loading. Concerning the level of the calculation, it seems to be overestimated demonstrating an unsatisfying calculation around the transition due to the simplistic phase kinetic model described by the hysteresis.

\section{Conclusion}

Experiments of tin from 8 to $15 \mathrm{GPa}$ were performed to investigate its polymorphic solid-solid transition under dynamic loading. For the first time, both velocity and temperature measurement has been performed at the interface allowing to improve our understanding of mixture phenomenon. Temperature measurements with an emissive layer have been achieved with sufficient precision to be compared to a model of phase transition. Velocity and temperature profiles display direct and reverse transition showing coherent singularities. These experimental results reveal clearly the existence of a hysteresis for the solidsolid transition during the loading and the release already studied by Mabire. The Mabire EOS associated to the SCG plasticity model provides an overall good estimate of the velocity profiles and their singularities. However, depending on the shock amplitude, its prediction of the temperature profile may be less satisfactory around the double structure of the direct $\beta$-bct transition. Hence, this underlines the limit of a model of a simple hysteresis, and the need for future improvements in terms of phase kinetics description.

\section{Acknowledgement}

The work was performed under the auspices of the DGA/ DO/UM NBC.

\section{References}

1. Mabire, C. and Hereil, P.-L., J. Phys. IV France 10, pp. 9-749-9-754, (2000).

2. Perez, M. and Costeraste, J., North-Holland, pp. 703706, (1987).

3. Seifter, A., et al., AIP Conf. Proc. 845, pp. 139-142, (2005).

4. Seifter, A., et al., Proc. High Speed Photography and Photonics, pp 18-23, SPIE paper \# :5580-125, (2005).

5. Poulsen P. and Hare D.E., UCRL-ID-146845, february 4, (2002).

6. Chauvin, C., et al., Dymat 2009, EDP Sciences, pp. 311-317, (2009).

7. Partouche D., Holtkamp D., Pelissier J., Taboury J. and Rouyer A., Shock Waves, 11, pp. 385-392, (2002).

8. Grover, R. G. and Urtiew, P., A., Journal of Applied Physics, vol. 45, no. 1, pp. 146-152, (1974).

9. Marsch, S. P., LASL Hugoniot Data, (1980)

10. Steinberg, D., UCRL-Ma-106439, February 13, (1996). 